\title{
Rat Bone Mesenchymal Stem Cell-Derived Exosomes Loaded with miR-494 Promoting Neurofilament Regeneration and Behavioral Function Recovery after Spinal Cord Injury
}

\author{
Wei Huang $\mathbb{D}$, ${ }^{1,2}$ Miaoman Lin $\mathbb{D}^{1},{ }^{1}$ Cunheng Yang $\mathbb{D},{ }^{1}$ Fumin Wang $\mathbb{D}^{1},{ }^{1}$ Meng Zhang $\mathbb{D}$, \\ Junxiao Gao ${ }^{1},{ }^{1}$ and Xiaobing Yu $\mathbb{1}^{1}$ \\ ${ }^{1}$ Department of Orthopaedics, Affiliated Zhongshan Hospital of Dalian University, Dalian 116001, China \\ ${ }^{2}$ Department of Orthopaedics, Dongguan Tungwah Hospital, Dongguan 523000, China \\ Correspondence should be addressed to Xiaobing Yu; yuxiaobing1976@hotmail.com
}

Received 14 June 2021; Revised 5 September 2021; Accepted 9 September 2021; Published 1 October 2021

Academic Editor: Dragan Hrnčić

Copyright (C) 2021 Wei Huang et al. This is an open access article distributed under the Creative Commons Attribution License, which permits unrestricted use, distribution, and reproduction in any medium, provided the original work is properly cited.

Exosomes (Exo) exhibit numerous advantages (e.g., good encapsulation, high targeting efficiency, and easy to penetrate the blood-brain barrier to the central nervous system). Exosomes are recognized as prominent carriers of mRNAs, siRNAs, miRNAs, proteins, and other bioactive molecules. As confirmed by existing studies, miR-494 is important to regulate the occurrence, progression, and repair of spinal cord injury (SCI). We constructed miR-494-modified exosomes (Exo-miR-494). As indicated from related research in vitro and vivo, Exo-miR-494 is capable of effectively inhibiting the inflammatory response and neuronal apoptosis in the injured area, as well as upregulating various anti-inflammatory factors and miR-494 to protect neurons. Moreover, it can promote the regeneration of the neurofilament and improve the recovery of behavioral function of SCI rats.

\section{Introduction}

Spinal cord injury (SCI) refers to a catastrophic event in spinal surgery, commonly leading to severe neurological and sensory dysfunction [1]. It still lacks effective treatment measures in clinical practice, imposing huge psychological and economic burden to patients and the country's medical services. Over the past few years, the powerful proliferation and differentiation potential of mesenchymal stem cells (MSCs) has achieved new progress for treating SCI [2]. However, the transplanted MSCs have numerous defects (e.g., low survival rate, carcinogenicity, and poor targeting) [3]. As proven by numerous studies, the therapeutic effect of MSCs may be primarily attributed to the secreted exosomes (Exo) [4].

Exos are small membrane vesicles originating from intracellular corpuscles, exhibiting a diameter of about 30$200 \mathrm{~nm}$, which are packed with various proteins, lipids, and RNA. These vesicles can be released into extracellular fluid, blood, and cerebrospinal fluid by cells of all living systems $[5,6]$. Under physiological and pathophysiological conditions, they are capable of transferring their cargo between source cells and target cells via extracellular fluid, blood, and cerebrospinal fluid, and they critically impact intercellular communication [7]. Compared with the transplanted MSCs, MSCs-Exo exhibit the advantages of long stay time in the body, low carcinogenicity, and high targeting efficiency, as well as low immune rejection and easy of passing through the blood-brain barrier. Thus, it acts as an excellent carrier of foreign small molecule drugs.

Exosomes are closely related to the occurrence, progress, and recovery of central nervous system injury [8-10]. After spinal cord injury, exosomes can regulate the autonomic regeneration function of neurons and, also, affect the remyelination of the spinal cord, the metabolic activity, and inflammatory environment around the lesions. Exosomes can also actively mobilize the internal regeneration ability of neurons and reduce the adverse factors of the 


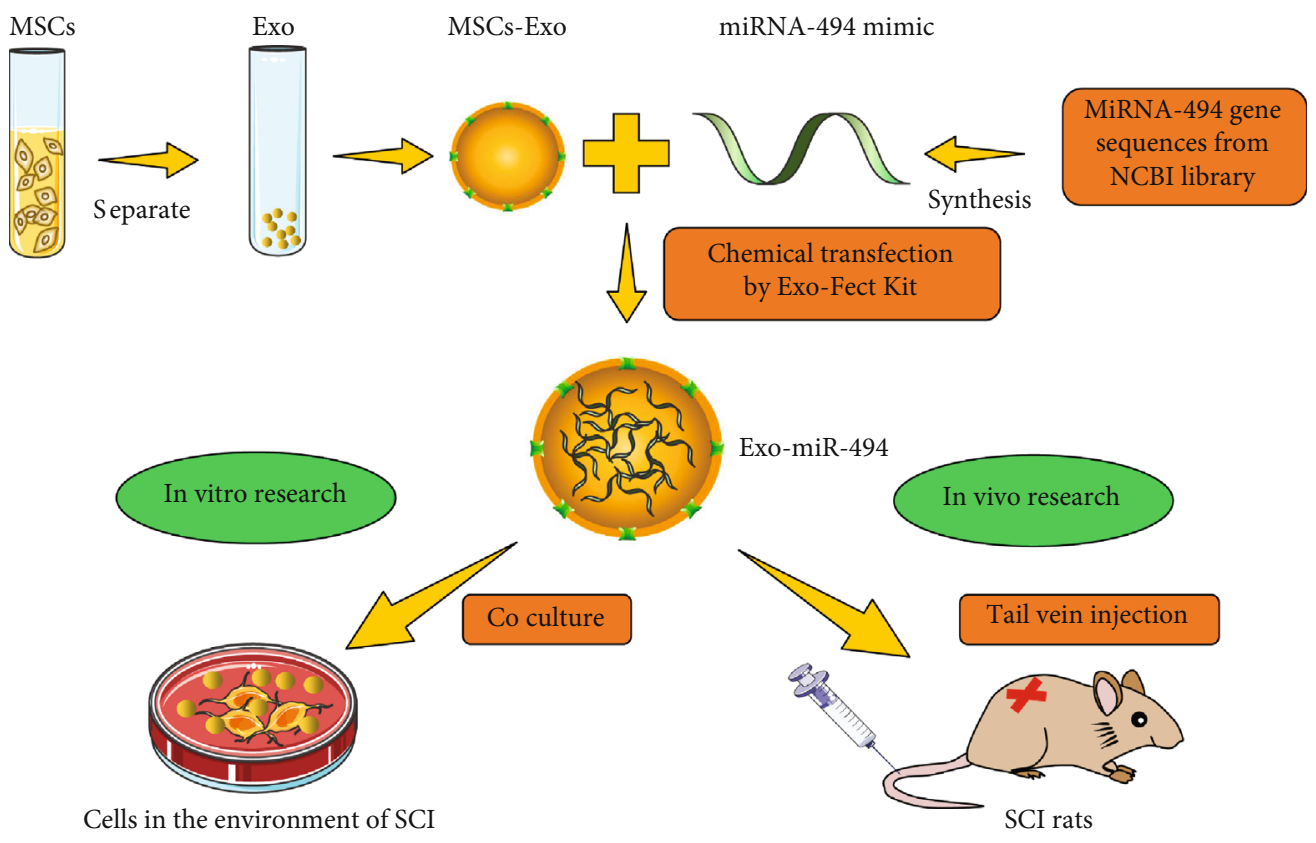

Figure 1: The flow chart of the whole experiment.

external surrounding microenvironment [11]. Studies have shown that bone marrow mesenchymal stem cell-exosomes can protect the injured spinal cord by inhibiting pericyte pyroptosis [12]. Furthermore, nanoscale exosomes secreted by stem cells have great potential in reducing apoptosis and inflammation, enhancing angiogenesis, and improving functional and behavioral recovery after spinal cord injury [13].

MicroRNAs (miRNAs) are small noncoding RNA molecules, capable of regulating various biological activities and profoundly impacting cell proliferation, differentiation, aging, death, neural activity, etc. miRNAs show a close relationship to the pathological processes of spinal cord injury (e.g., inflammatory reaction, demyelination, oxidative stress, and neuronal apoptosis) [14].

A large number of miRNAs have been found in the central nervous system of mammals, which play an important role in neurogenesis, development, and regeneration [15]. As reported by $\mathrm{Xu}$ et al., in the treatment of spinal cord injury, the overexpressions of miR-124 and miR-133b promote the differentiation of neural stem cells $[16,17]$. It has been reported that miR-494 can reduce autophagy and apoptosis of neurons after SCI by targeting IL-13 [18]. In addition, Wang et al. suggested that miR-494 is capable of regulating the effects of the Nogo/NgR signaling pathway on astrocyte proliferation and synaptic remodeling after SCI [19].

Though miR-494 exhibits a strong repair ability for SCI, the amount of secretion by living cells is low, and the efficiency of in vivo transportation is limited, thereby adversely affecting its therapeutic effect. This study planned to load exogenous miR-494 into exosomes through reagent transfection to determine whether exosomes can effectively deliver miR-494 to the spinal cord lesion, as well as verify the repair effect of Exo ${ }^{\text {miR-494 }}$ on SCI rats.

\section{Methods and Materials}

2.1. Experimental Design. The whole experiment is divided into three parts, "construction of Exo ${ }^{\text {miR-494 }}$ /Exo-miR-494," "in vitro research," and "in vivo research." As shown in Figure 1, firstly, we used the kit to extract the exosomes from rat MSCs and then designed and synthesized the miR-494 mimics according to the gene sequence of miR-494. Next, we used the Exo-Fect ${ }^{\mathrm{TM}}$ transfection kit to transfect miR494 into the MSCs-Exo and got the Exo ${ }^{\mathrm{miR}-494}$. In the second part, we cocultured the constructed Exo-miR-494 with dorsal root ganglions (DRGs) and rat NR8383 macrophages in the spinal cord injury environment to study whether it has antiapoptotic and anti-inflammatory effects. Finally, we conducted animal experiments. We injected Exo ${ }^{\text {miR-494 }}$ into SCI rats by tail vein injection to further study its effects on motor and behavioral functions of SCI rats.

2.2. Animals. The experimental procedures were approved by the Affiliated Zhongshan Hospital of Dalian University Science Review Committee and Animal Research Ethics Review Committee and conducted in line with the National Institutes of Health Guide for the Care and Use of Laboratory Animals.

First, we will use 2-week-old SD rats to extract bone MSCs. Then, in order to ensure that the grouping is statistically significant, we also need 6 SCI rats for the in vitro organ imaging experiment. Next, we need 6 normal healthy rats and 6 SCI rats for the drug toxicity test (the same animal can take the lung and liver at the same time, as well as the determination of lactate dehydrogenase, superoxide dismutase, and alanine aminotransferase in the liver).

Next, we carried out the animal experiment to verify the therapeutic effect of Exo ${ }^{\mathrm{miR}-494}$ on spinal cord injury. On the 
whole, grouping of animal experiments, i.e., the Control group (rats were treated with PBS after SCI), Sham group (rats were treated with PBS after sham operation), ExomiR-con group (rats were treated with pure exosome after SCI), and Exo-miR-494 group (rats were treated with ExomiR-494 after SCI). In order to ensure the statistical significance of each experiment and ensure that the number of rats in each group is 6, we divided the animals into the Control group $(n=6)$, Sham group $(n=6)$, Exo-miR-con group $(n=6)$, and Exo-miR-494 group $(n=6)$ to form a large group. In this way, we need a large group of animals to determine the relative expression of miR-494 and growthassociated protein-43(GAP-43) in vivo. Similarly, we need a large group of animals for the hematoxylin-eosin (HE) experiment, immunofluorescence analysis experiment, locomotor capacity assessment experiment, Paw Withdraw Thermal Latency (PWTL) experiment, and hind leg neurophysiological experiment. Statistically, we need 22 -weekold SD rats, 6 normal healthy rats, 42 sham rats, and 138 SCI rats (excluding the rats with SCI model failure or death, continue to supplement the above conditions, and all animal numbers are confirmed by the Animal Research Ethics Review Committee).

2.3. Extraction and Identification of Bone Marrow MSCs. Firstly, male SD rats (80-100 g) of 2 weeks old were prepared, the whole bone marrow culture method was used to extract MSCs, and then, MSCs were cultured in primary culture until the cell density reached $90 \%$. Subsequently, the serum-free FBS (Gibco ${ }^{\mathrm{TM}}$, USA) was used for $48 \mathrm{~h}$ expansion culture in the complete medium to obtain about $300 \mathrm{~mL}$ of stem cell culture supernatant for subsequent experiments.

Next, we used flow cytometry to identify the expression of the bone marrow MSC marker protein. In short, firstly, MSCs were digested with trypsin and mixed with $100 \mu \mathrm{L}$ PBS, and then, $10 \mu \mathrm{L}$ of CD29, CD45, and CD90 antibodies (eBioscience, USA) was added. The mixture was wrapped with tin foil and incubated at $4^{\circ} \mathrm{C}$ for $30 \mathrm{~min}$. After incubation, centrifuge for $5 \mathrm{~min}$, clean 3 times, and resuspend MSCs with $500 \mu \mathrm{L}$ PBS. Finally, the marker protein was detected by flow cytometry (Cytek, USA), and the data were processed by FlowJo 8.7 software.

\subsection{Preparation and Identification of Exosomes Derived from} Bone Marrow MSCs. With the kit method, the exosomes were separated from the collected cell supernatant. In brief, the cell supernatant was placed at $4^{\circ} \mathrm{C}$ and then centrifuged at $3000 \mathrm{~g}$ for $10 \mathrm{~min}$ to remove excess cell debris. Subsequently, it was centrifuged to remove impurities. GS ${ }^{\mathrm{TM}}$ Exosome Isolation Reagent (for serum or plasma, Geneseed Biotech Co., China) was added to the supernatant, and the instructions (Supplement 1) were complied with to extract exosomes from the extracellular supernatant. In summary, $500 \mu \mathrm{L}$ of the rat supernatant was extracted, and $1 / 3$ times the volume of the supernatant was added to the solution A. And the mixed solution was left to stand at $4^{\circ} \mathrm{C}$ for $15 \mathrm{~min}$. At ambient temperature, centrifugation was conducted again at $13,000 \mathrm{~g}$ for $10 \mathrm{~min}$, and the supernatant was aspirated; the solution was centrifuged at $13,000 \mathrm{~g}$ for
$30 \mathrm{~s}$, and the residual supernatant was aspirated. Lastly, the identical volume of $\mathrm{B}$ solution was added as Reagent $\mathrm{A}$, and the solution was mixed by pipetting and centrifuged at $13,000 \mathrm{~g}$. Finally, the supernatant was aspirated and removed; afterwards, we used bicinchoninic acid (BCA) to detect the protein concentration in the exosome and then resuspended it in PBS with a total protein concentration of $10 \mu \mathrm{g} / \mu \mathrm{L}$ [20]. The resulting precipitate was an exosome, which was stored at $-80^{\circ} \mathrm{C}$. The mentioned method was repeated to extract all the exosomes in the supernatant of $500 \mathrm{~mL}$ for subsequent cell and animal experiments.

Next, we used the following methods to identify exosomes.

2.4.1. Transmission Electron Microscopy (TEM). Take out $5 \mu \mathrm{L}$ exosomes, add $5 \mu \mathrm{L}$ PBS, then add the mixture to the copper wire, and stand for $1 \mathrm{~min}$; next, $10 \mu \mathrm{L}$ phosphotungstic acid was added to the copper mesh and stood for $1 \mathrm{~min}$ to remove the liquid suspended on the surface (refer to Supplement 2 for specific methods). Finally, the imaging was detected by $80 \mathrm{kV}$ transmission electron microscopy (Hitachi, JAPAN).

2.4.2. Nanoparticle Tracking Analysis (NTA). Take $10 \mu \mathrm{L}$ exosomes and add $990 \mu \mathrm{L}$ PBS which was mixed evenly, and nanoparticle tracking analysis instrument (Particle Metrix, Germany) was used to detect the sample. Besides, we detected the marker proteins CD9 and CD63 of exosomes by western blot (WB) analysis (see "WB Analysis" for specific methods).

2.5. Preparation of miR-494 Mimic and Negative Controls (NC). By complying with the instructions, miR-494 mimics and miR-494 NC were synthesized by Invitrogen (GenePharma, Shanghai, China), treated with diethyl pyrocarbonate (DEPC), and then placed and stored at a low temperature and in a dark place. The oligonucleotides used in this sequence are shown in Table 1.

2.6. MiR-494 Was Loaded into Exosomes by Chemical Transfection. As mentioned above [21], we used Exo-Fect ${ }^{\mathrm{TM}}$ (System Biosciences, Palo Alto, USA) to load miR-494. First, miR-494 (100-300 pmol), exosomes $(200 \mu \mathrm{g}$ in $50 \mu \mathrm{L})$, and Exo-Fect were mixed and incubated at $37^{\circ} \mathrm{C}$ for $20 \mathrm{~min}$ (for the Control group, only miR-494 and exosomes were mixed without adding Exo-Fect), then ExoQuick-TC reagent (System Biosciences, Palo Alto, USA) was used to culture the mixture on ice for $20 \mathrm{~min}$. Finally, exosomes/Exo ${ }^{\mathrm{miR}-494}$ were centrifuged at $13,000 \times \mathrm{g}$ for $5 \mathrm{~min}$ to collect and resuspend in phosphate buffer saline (PBS, Gibco ${ }^{\mathrm{TM}}$, USA) for reverse transcription (qPCR).

\subsection{Quantitative Determination and Antidegradation Test of Exo ${ }^{\text {miR-494 }}$}

2.7.1. Exo ${ }^{\text {miR-494 }}$ Loading Capacity. $5^{\prime}-32 \mathrm{P}$ were used to label miR-494. The loading capacity of exosomes was determined by the radioactivity in the supernatant and then quantified with a Packard instant imager. The loading rate was calculated using the following equation: the $\mathrm{miR}-494$ loading rate $=(\mathrm{CPM}$ in pellet $/ \mathrm{CPM}$ in supernatant $) \times 100$. Moreover, 
TABLE 1: Synthesized miR-494 mimics and negative control (NC).

\begin{tabular}{lc}
\hline Gene & Sequence $\left(5^{\prime}\right.$ to $\left.3^{\prime}\right)$ \\
\hline miR-494 mimic & UGAAACAUACACGGGAAACCUC \\
miR-494 mimic NC & UUCUCCGAACGUGUCACGU \\
\hline
\end{tabular}

the loading capacity of Exo ${ }^{\text {miR-494 }}$ was calculated by the aforesaid formula.

2.7.2. Exo ${ }^{\text {miR-494 }}$ Antidegradation Test. Exo ${ }^{\text {miR-494 }}$ were incubated with $500 \mu \mathrm{L}$ RNase A with various concentrations $\left(0.01 \mu \mathrm{g} / \mathrm{mL}\right.$, Solarbio, USA). After being incubated at $37^{\circ} \mathrm{C}$ for $0,30,60$, and $90 \mathrm{~min}$, naked $\mathrm{miR}-494 \mathrm{mimic}$ was classified as the control. Moreover, the undegraded miR-494 was quantitatively analyzed by RT-PCR.

2.8. Apoptosis Test. Rat dorsal root ganglion cells (DRGs) were obtained from the cell bank center of the Shanghai Academy of Biological Sciences (SIBS) and were seeded in a 6-well plate with $1 \times 10^{6}$ cells/well. When DRGs fused more than $90 \%$, the SCI environment was simulated with $10 \mathrm{mg}$ lipopolysaccharide (LPS, YT1319, YITA, China) and hypoxia $\left(2 \% \mathrm{O}_{2}\right)$ for $24 \mathrm{~h}$. Referring to previous studies [20], next, the experimental groups were as follows $(n=3)$ : Control group (0.5 mL PBS), Exo-miR-Con group (100 $\mu \mathrm{g}$ pure exosomes in $0.5 \mathrm{~mL} \mathrm{PBS}$ ), and Exo-miR-494 group $\left(100 \mu \mathrm{g} \mathrm{Exo}{ }^{\text {miR-494 }}\right.$ in $0.5 \mathrm{~mL}$ PBS). After 24 hours of coculture, DRGs were washed twice with PBS, and the survival of the cells was evaluated by an acridine orange endothelium bromide (AOEB) staining kit (Jizhi Biochemical Technology, Shanghai), and apoptotic proteins (Caspase-3, Bax, and $\mathrm{Bcl}-2$ ) were also extracted for western blotting as previously described [22].

2.9. Enzyme-Linked Immunosorbent Assay (ELISA). The culture, seed plate, and grouping of rat NR8383 macrophages (ATCC, SIBS, China) were the same as DRGs. The experimental groups were as follows $(n=3)$ : Control group (0.5 mL PBS), Exo-miR-Con group (100 $\mu \mathrm{g}$ pure exosomes in $0.5 \mathrm{~mL}$ PBS), and Exo-miR-494 group (100 $\mu \mathrm{g}$ Exo ${ }^{\mathrm{miR}-494}$ in $0.5 \mathrm{~mL}$ PBS). After 48 hours of coculture, the expression of inflammatory factors was detected by ELISA kits: rat TNF- $\alpha$, IL-6, IL-8, and IL-10 ELISA kit (Thermo Scientific, Shanghai, China).

2.10. Establishment of SCI Rat Model and Experimental Grouping. The rats were anesthetized with $4 \%$ sodium pentobarbital ( $50 \mathrm{mg} / \mathrm{kg}$; Beyotime Biotechnology, China). At the $\mathrm{T} 10$ level, a $2 \mathrm{~cm}$ incision was achieved to expose the spinal skeletal muscle in a sterile environment. Except for the Sham group, the T10 segment was impacted by the spinal cord injury percussion apparatus (Yuyan Instrument, China) in the other groups. The weight of the metal rod reached $25 \mathrm{~g}$, and the height was $50 \mathrm{~mm}$. Subsequently, involuntary twitch and tail swing were identified in both hind limbs of all rats, demonstrating that the injury reached the standard of the spinal cord injury model. In the Sham group, the above operations were performed without spinal cord injury. After suturing the wound, the rats after opera- tion recovered in a warm incubator. In addition, rats in the Sham group underwent the above operations without spinal cord injury.

After operation, the animals were urinated three times a day until they could urinate by themselves. Tail-vein administration was performed per $24 \mathrm{~h}$ for 7 consecutive days after establishment of the SCI model. To be specific, the dosage was as follows: Control group and Sham group were administered with $1 \mathrm{~mL}$ PBS, while the Exo-miR-con group with $100 \mu \mathrm{g}$ pure exosomes (diluted in $1 \mathrm{~mL}$ PBS), and the ExomiR-494 group was treated with $100 \mu \mathrm{g}$ Exo ${ }^{\text {miR-494 }}$ (diluted in $1 \mathrm{~mL}$ PBS).

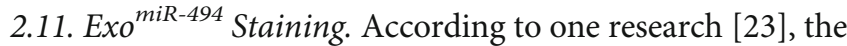
dye working solution was prepared. In short, dilute the dye storage solution with diluent $\mathrm{C}$ reagent (UR52303, Umibio, China), prepare the PKH67 dye working solution with the concentration of $100 \mathrm{~nm}$, add $10 \mu \mathrm{L}$ PKH67 dye working solution to the Exo ${ }^{\mathrm{miR}-494}$, mix well, and culture at room temperature for $5 \mathrm{~min}$. The reaction between Exo ${ }^{\mathrm{miR}-494}$ and PKH67 dye working solution was terminated for $2 \mathrm{~min}$ by adding $500 \mu \mathrm{L} 1 \%$ bovine serum albumin (BSA). Afterwards, the volume of the mixture was added to $25 \mathrm{~mL}$ and then centrifuged for $45 \mathrm{~min}$ at $120,000 \mathrm{~g}$. The supernatant was discarded, and the residue was suspended in PBS. The final precipitate was PKH67-Exo ${ }^{\text {miR-494. }}$.

2.12. Drug Toxicity Test of Exo ${ }^{\text {miR-494 }}$ to the Lung and Liver. After 24 hours of treatment with Exo ${ }^{\mathrm{miR}-494}$, the lung and liver tissues of rats in the Exo-miR-494 group were taken out for HE staining and compared with those of healthy rats. Simultaneously, the activities of lactate dehydrogenase (LDH), superoxide dismutase (SOD), and alanine aminotransferase (ALT) in the healthy rat liver were measured by the enzyme labeling method. Firstly, we extract $20 \mu \mathrm{g}$ liver homogenate, and PBS were used to lyse cells. Then, three enzyme assay kits (Wanlei Bio, China) were added to the cell culture medium, the LDH activity, ALT activity, and SOD activity according to the instructions (Supplement 4, 5, 6). The following are the specific detection methods.

2.12.1. LDH Detection (Visible Light Colorimetry). First, take 10-50 $\mu \mathrm{L}$ rat liver homogenate. Then, add the $\mathrm{LDH}$ kit, mix well, and place in a water bath at $37^{\circ} \mathrm{C}$ for $15 \mathrm{~min}$; finally, set the wavelength to $440 \mathrm{~nm}$ and the optical path to $1 \mathrm{~cm}$ and measure the absorbance OD value of each tube with an ultraviolet spectrophotometer (Shimadzu, Japan).

2.12.2. Calculation Formula of Tissue LDH Activity. $1.5 \%$ is produced in the reaction system when each gram of tissue protein reacts with the matrix at $37^{\circ} \mathrm{C}$ for $15 \mathrm{~min} ; 1 \mu \mathrm{mol}$ pyruvate is 1 unit (U).

2.12.3. SOD Detection (Hydroxylamine Method). First, take $20 \mu \mathrm{L}$ rat liver homogenate. Then, add the SOD kit, mix well, and place at room temperature for $10 \mathrm{~min}$; finally, set the wavelength to $550 \mathrm{~nm}$ and the optical path to $1 \mathrm{~cm}$ and measure the absorbance OD value of each tube with an ultraviolet spectrophotometer. 
2.12.4. Calculation of Total SOD Activity in Tissue Homogenate. When the inhibition rate of SOD per mg tissue protein in $1 \mathrm{~mL}$ reaction solution reaches $50 \%$, the corresponding SOD amount is one SOD activity unit (U).

2.12.5. ALT/GPT Detection (Visible Light Colorimetry). Mix the ALT kit with $50 \mu \mathrm{L}$ rat liver tissue; place it at room temperature for $5 \mathrm{~min}$, wavelength $505 \mathrm{~nm}$, optical path $1 \mathrm{~cm}$, and zero with double distilled water; and measure the absorbance OD value of each tube. Check the standard curve to obtain the corresponding ALT/GPT activity unit.

Moreover, after 48 hours of administration, miR-494 were extracted for further quantitative RT-PCR analysis.

2.13. Total RNA Isolation and Quantitative Real-Time Polymerase Chain Reaction ( $q R T-P C R)$. RNA extraction kits (Solarbio, China) were adopted to extract total RNA from cells and spinal cord lesion tissues. To be specific, miRNA extraction kits (Solarbio, China) were used for miR-494 in exosomes or cells, target mRNA or miR-494 acted as templates, and reverse transcription kits (Solarbio, China) were employed for reverse transcription into cDNA. Relative quantification of RNA expression levels was performed on an ABI 7500 Real-Time PCR system (Applied Biosystems, Carlsbad, CA). Furthermore, the $2^{-\Delta \Delta \mathrm{Ct}}$ method was employed for relative quantification, and U6 was the internal reference gene (refer to Supplement 3 for specific methods). Table 2 lists all the primers applied in the experiment.

2.14. WB Analysis. RIPA buffer was employed to extract the total exosomes, protein of cells, and spinal cord tissue, and WB experiments were performed by complying with the manufacturer's protocol. Primary antibodies include CD63, CD9, GAPDH, $\beta$-actin, Caspase-3, Bax, Bcl-2, and GAP-43 (rabbit, Abcam, UK).

2.15. HE Staining of Spinal Cord Tissue. After 4 weeks of administration, rats in each group were anesthetized with $4 \%$ sodium pentobarbital $(50 \mathrm{mg} / \mathrm{kg}$ ). Then, $1 \mathrm{~cm}$ spinal cord segments (including injury center) were stained with $\mathrm{HE}$ staining. The center of the lesion was the most severe transverse section.

2.16. Immunofluorescence Analysis. After 4 weeks of administration, the longitudinal spinal cords of rats in each group were cut into frozen sections at $0.02 \mathrm{~mm}$ intervals. Subsequently, the sections were permeabilized in $0.5 \%$ Tween solution and blocked with $5 \%$ bovine serum, combined with mouse neurofilament (NF-H) antibody (1:1000, Abcam, UK) and rabbit glial fibrillary acidic protein (GFAP) antibody $\left(1: 100\right.$, Abcam, UK) overnight at $4^{\circ} \mathrm{C}$. Afterwards, the spinal cord sections were incubated with DAPI ( $1: 1000$, Invitrogen) and goat anti-rabbit IgG $(\mathrm{H}+\mathrm{L})$ secondary antibody ( $1: 300$, Invitrogen) for $1 \mathrm{~h}$, and the image was captured under a confocal fluorescence microscope (Leica Microsystems, Germany).

Furthermore, we use ImageJ software (V1.8.0.112) to measure the relative expression of NF and GFAP by average fluorescence intensity. First, we use the ImageJ software to open the picture of single fluorescence (NF or GFAP), then
TABLe 2: Primers used for RT-qPCR.

\begin{tabular}{lc}
\hline Gene & Sequence $\left(5^{\prime}\right.$ to $\left.3^{\prime}\right)$ \\
\hline miR-494 & Forward CATAGCCCGTGAAACATA CACG \\
& Reverse GTGCAGGGTCCGAGGT \\
U6 & Forward CGCTTCGGCAGCACATATACTA \\
& Reverse GCGAGCACAGAATTAATACGAC \\
\hline
\end{tabular}

adjust the threshold (software default), select the appropriate threshold algorithm, and detect and analyze the result. The average fluorescence intensity formula is as follows: average fluorescence intensity $=$ total fluorescence intensity of the area/the area.

2.17. Locomotor Capacity Assessment. The locomotor capacity of hind limbs of all rats was assessed by Basso, Beattie, and Bresnahan (BBB) locomotor rating scale. 0 means no motor activity, and 21 means normal motor activity. The mentioned results were observed by two independent researchers at 1 day before and $1,7,14,21$, and 28 days after injury.

2.18. Paw Withdraw Thermal Latency (PWTL). On days 0, 7, 14,21 , and 28 after injury, the thermal sensitivity of rats was detected by PWTL. The radiant heat source (Techman Company, China) was placed in the middle of the posterior surface of the rat sole. After thermal stimulation, the rats' hind paw moved or $20 \mathrm{~s}$ later, the thermal stimulator would automatically close to avoid tissue damage, and the pain threshold time was recorded. The effects of $50 \%$ radiant heat intensity and 6-8 s heat block latency on the normal pain threshold were observed.

2.19. Hind Leg Neurophysiological Experiment. After 1 month of treatment, rats in each group underwent the experiment. In brief, $4 \%$ sodium pentobarbital $(50 \mathrm{mg} / \mathrm{kg})$ were adopted to anesthetize animals. Transcranial electrical stimulation was applied with pulse, and a SEN-7301 constant current isolation stimulator (Nihon Kohden Corp., Japan) was employed for $1 \mathrm{~ms}$ at $9 \mathrm{mV}$. Two $30 \mathrm{~g}$ stainless steel stimulation electrodes (Nihon Kohden Corp., Japan) were implanted subcutaneously into the hind limbs of rats to record motor evoked potentials (MEPs) of rat hind limbs until we obtained 3 5 stable and repeatable potentials, and we continuously recorded the amplitude of MEP for 3 times.

2.20. Statistical Analysis. SPSS 21.0 statistical software was used (IBM Corp., Armonk, NY, USA). Data were compared by performing two-tailed Student's $t$-test or one-way analysis of variance and Tukey's post hoc test. $p<0.05$ was considered to be statistically significant.

\section{Results}

3.1. Characterization of Exo and Exo ${ }^{\text {miR-494 }}$. As mentioned above, we successfully isolated MSCs. Flow cytometry showed that the isolated BMSCs highly expressed CD29 (99.9\%), CD90 (99.2\%), and low expressed CD45 (0.37\%), which were in accordance with the characteristics of rat BMSCs (Figure 2(a)). Next, we extracted considerable Exo 

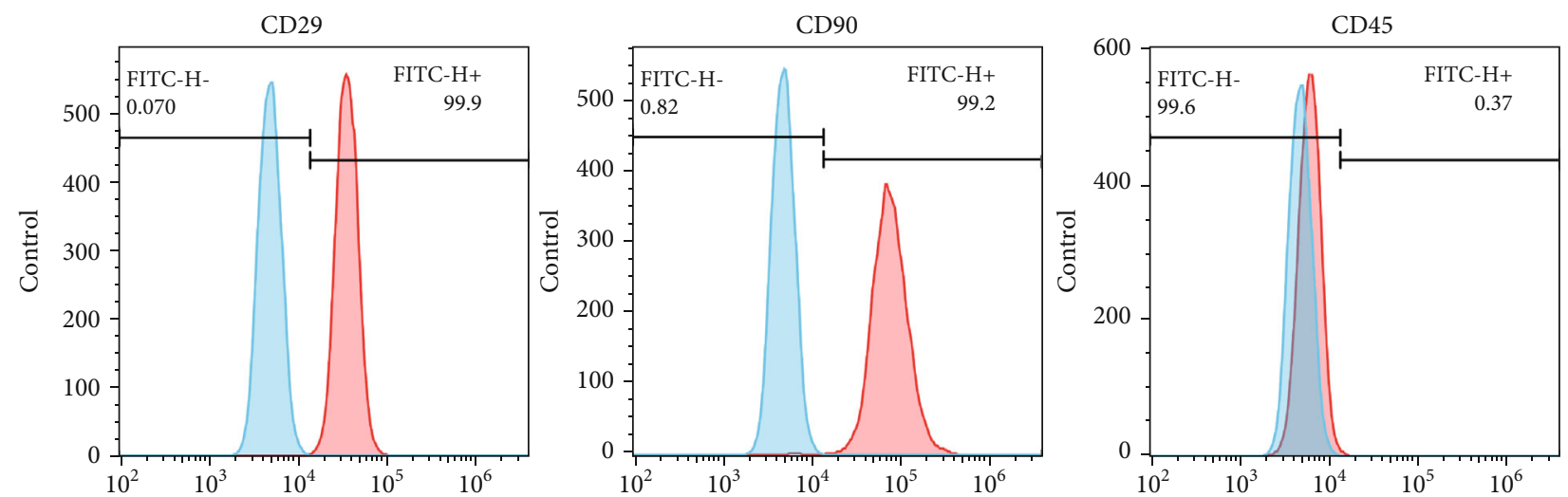

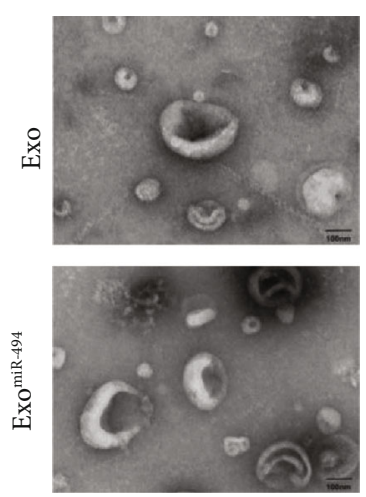

(b)

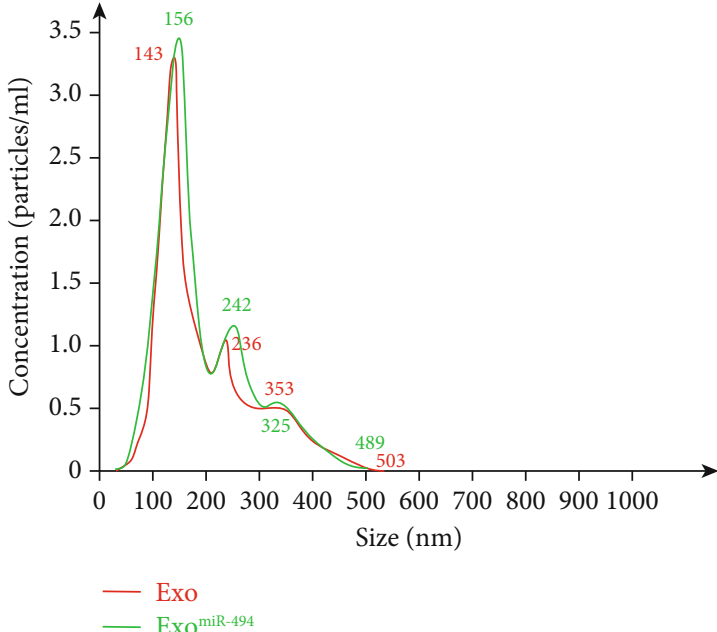

(c)

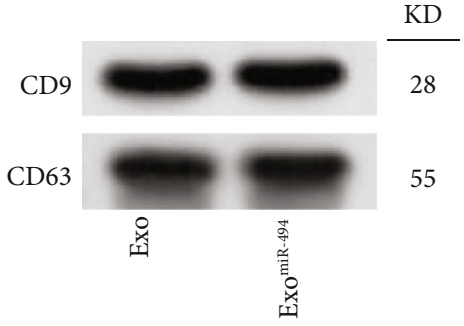

(d)

Figure 2: Characterization of BMSC-Exo and Exo ${ }^{\text {miR-494 }}$. (a) Expression of BMSC markers (CD29, CD45, and CD90) was identified by flow cytometry. (b) Morphological observation of Exo and Exo ${ }^{\text {miR-494 }}$ by transmission electron microscopy (indicated by yellow arrow). Scale bar $=100 \mathrm{~nm}$. (c) Nanoparticle tracking analysis of Exo and Exo ${ }^{\text {miR-494 }}$. (d) The BMSC-Exo marker proteins (CD9, CD63) were analyzed using western blot analysis.

from the supernatant of rat bone marrow MSCs by the kit method and compared Exo with the subsequent construction of Exo ${ }^{\text {miR-494 }}$. First, we observed Exo/Exo ${ }^{\text {miR-494 }}$ under TEM by negative staining, and TEM results showed that many Exos and Exo ${ }^{\text {miR-494 }}$ (the yellow arrow indicates Exos or Exo ${ }^{\text {miR-494}) ~ h a d ~ a ~ t y p i c a l ~ s a u c e r-l i k e ~ s t r u c t u r e ~ w i t h ~ a ~ s i z e ~}$ of approximately 30-200 nm (Figure 2(b)), and NTA also showed that the diameters of Exo and Exo ${ }^{\text {miR-494 }}$ were concentrated at about $150 \mathrm{~nm}$ (Figure 2(c)), which showed that the incorporation of miR-494 did not affect the diameter or morphology of Exo. Moreover, the WB band also showed that miR-494 did not affect the expression of Exo-labeled proteins CD9 and CD63 (Figure 2(d)).

3.2. Exosome Loading miR-494 Has Good Loading Rate and Stability. According to existing studies [21], we loaded miR-494 into exosomes by Exo-Fect. The results of absolute quantitative RT-PCR showed that the exosomes of the ExoFect group contained more miR-494 molecules, with the number of about $2.26 * 10^{14} / \mu \mathrm{g}$ exosomes (Figure 3(a)), and the sample loading rate/transfection efficiency of miR-
494 is about $38.24 \%$ (Figure 3(b)). Interestingly, the number of molecules in the Control group (untransfected) also had $1.12 * 10^{11} / \mu$ g exosomes, which may be due to the fact that exosomes can also actively take up some miRNAs in very small amounts (Figure 3(b)).

Furthermore, in order to test the stability of Exo ${ }^{\text {miR-494 }}$ in the circulatory system, we treated naked miR-494 and Exo ${ }^{\text {miR-494 }}$ with RNase A. The results of RT-PCR showed that the naked miR-494 was degraded gradually with time, and the residual amount of miR-494 was less than $15 \%$ at $90 \mathrm{~min}$, while the residual amount of miR-494 in Exo ${ }^{\mathrm{miR}-494}$ was still $44.8 \pm 2.1 \%$ (Figure 3 (c)). These results showed that exosomes had a good drug loading effect and could effectively prevent the degradation of miRNAs in body fluid.

3.3. Therapeutic Effect of Exo ${ }^{\text {miR-494 }}$ on DRGs In Vitro. Existing studies showed that MSC-derived pure exosomes or pure miR-494 could inhibit neuronal apoptosis [18]. After LPS/hypoxia treatment, the DRGs were treated with PBS, $\mathrm{Exo}^{\mathrm{miR}-\mathrm{con}}$, and Exo ${ }^{\mathrm{miR}-494}$. Live/dead cell double staining showed that the DRG survival rate of the Control group 


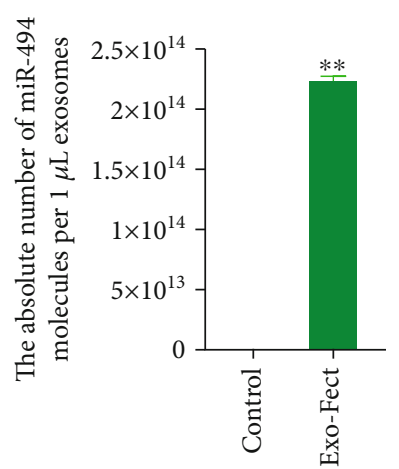

(a)

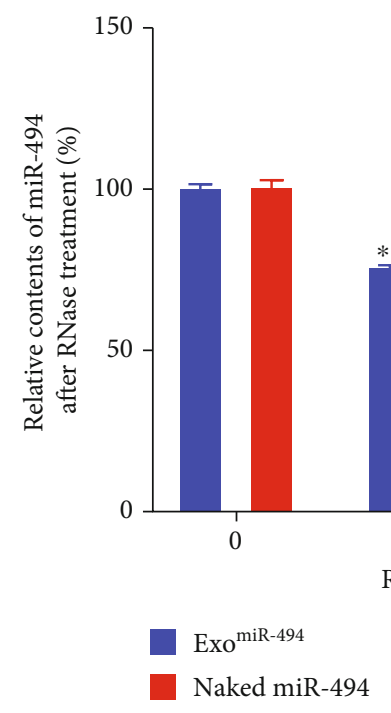

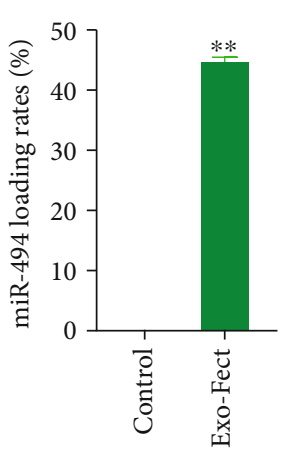

(b)

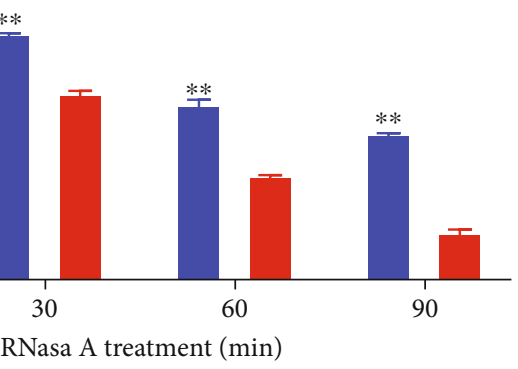

(c)

FIgURE 3: Exosome loading miR-494 by Exo-Fect. (a) The absolute number of miR-494 molecules in exosomes by absolute quantitative realtime PCR. $n=3$, data are represented as mean $\pm \mathrm{SD},{ }^{* *} p<0.01$ vs. Control. (b) The loading rates of miR-494 in Exo were presented. $n=3$, data are represented as mean $\pm \mathrm{SD},{ }^{* *} p<0.01$ vs. Control. (c) Relative contents of naked miR-494 and Exo ${ }^{\text {miR-494 }}$ after RNase A treatment. $n=6$, data are represented as mean $\pm \mathrm{SD},{ }^{*} p<0.05$ and ${ }^{* *} p<0.01$ vs. naked miR-494.

was significantly decreased, only $46.35 \%$, while the survival rate of the Exo-miR-494 group was as high as $80.1 \pm 2.5 \%$ (Figures 4(a) and 4(b)); In addition, the Exo-miR-con group also had a survival rate of $58.2 \pm 2.6 \%$, which was attributed to the antiapoptotic effect of the exosome alone (Figures 4(a) and $4(\mathrm{~b}))$. The WB trend of apoptotic protein was the same as the cell survival rate (Figure 4(c)). The Caspase- 3 and Bax in the Exo-miR-con group and the Exo-miR-494 group were significantly lower than those in the Control group (Figure $4(\mathrm{~d})$ ). On the contrary, the antiapoptotic protein $\mathrm{Bcl}-2$ in the Exo-miR-con and Exo-miR-494 groups was significantly higher than that in the Control group (Figure 4(d)), because both exosome and miR-494 have antiapoptotic effects, which may be coordinated with each other and conform to the cell survival trend.

3.4. Exo ${ }^{\text {miR-494 }}$ Can Promote the Polarity Transformation of Macrophages In Vitro and Inhibit Inflammatory Reaction. MSC-derived exosomes can regulate the polarization of macrophages and reduce the inflammatory response after SCI $[24,25]$. Next, we studied the relationship between
Exo ${ }^{\text {miR-494 }}$ and inflammatory response. The alveolar macrophages of NR8383 rats were treated with LPS/hypoxia for $24 \mathrm{~h}$ and then cocultured with miR-494, Exo ${ }^{\text {miR-con, }}$ and Exo ${ }^{\text {miR-494 }}$ for $48 \mathrm{~h}$. The expression of MI and M2 markers was detected by ELISA. As revealed from the results, Exo ${ }^{\text {miR-con }}$ or Exo ${ }^{\text {miR-494 treatment significantly }}$ decreased the expression of M1 polarization-related proinflammatory cytokines (TNF-6 and IL- $\alpha$ ), but the difference between the two groups was not obvious (Figure 4(e)). Meanwhile, Exo ${ }^{\text {miR-con }}$ or Exo ${ }^{\text {miR-494 }}$ treatment also significantly increased the expression of M2 polarization-related anti-inflammatory cytokines (IL-8 and IL-10) in Figure 4(f).

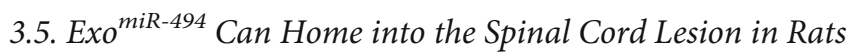
by Tail Vein Injection. Next, animal experiments were performed. The DIR iodide-labeled Exo ${ }^{\text {miR-494 }}$ were injected into SCI rats via the tail vein, and its distribution in the organs and spinal cord was observed under the animal fluorescence imaging system. As revealed from the results, $3 \mathrm{~h}$ imaging results showed that Exo ${ }^{\text {miR-494 }}$ showed relatively high fluorescence intensity in the lung and liver, which was 
Control

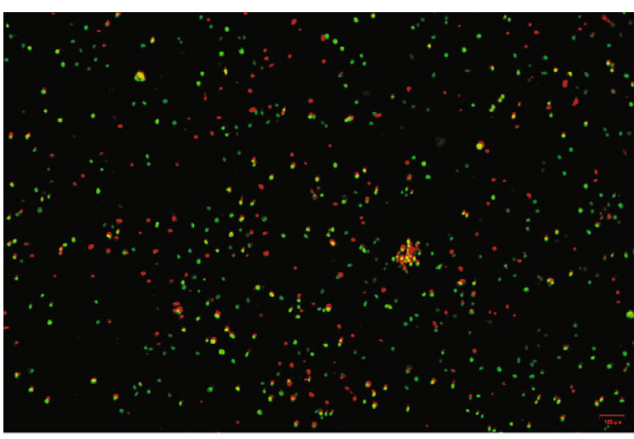

Exo-miR-494

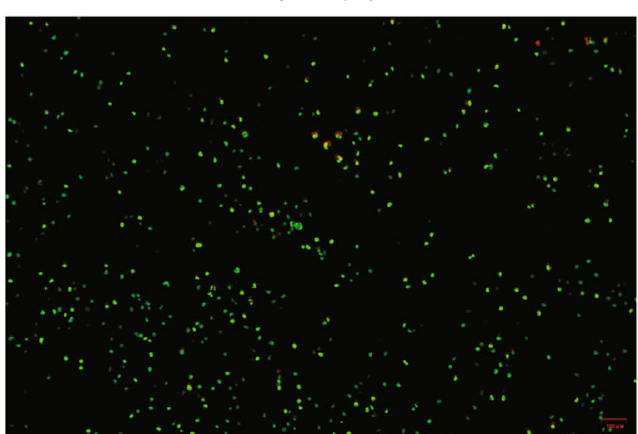

(a)

(b)

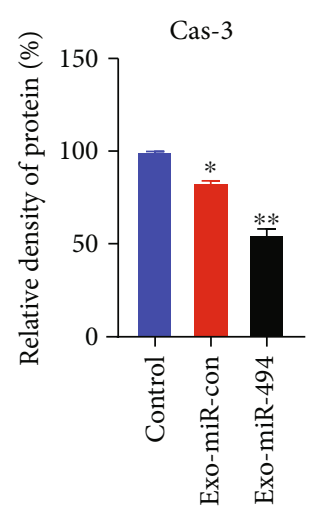

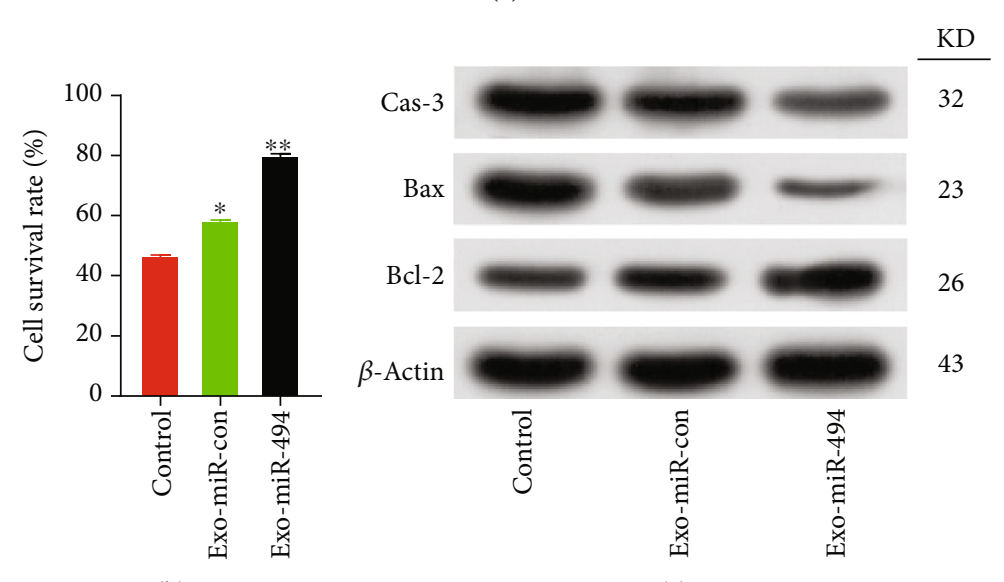

Exo-miR-con
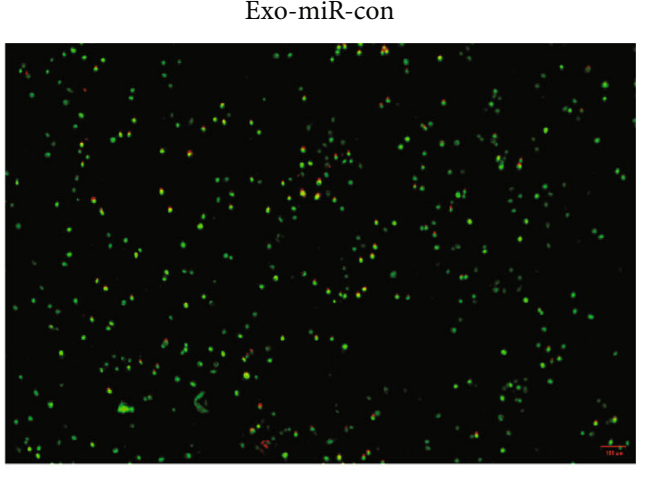

\section{.}

(1)
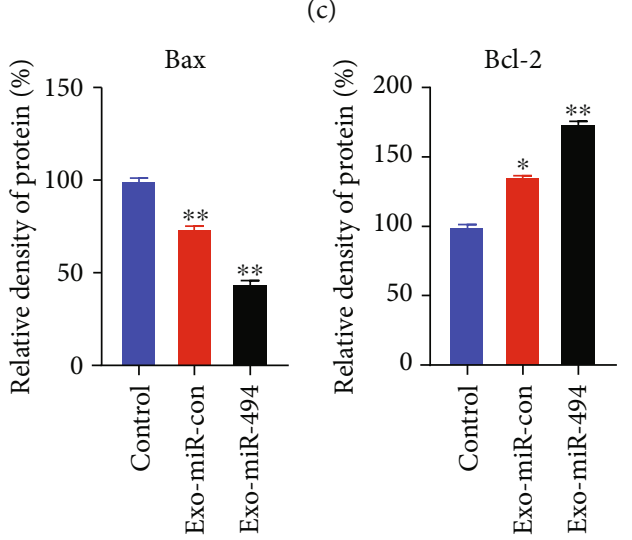

(d)

Figure 4: Continued. 


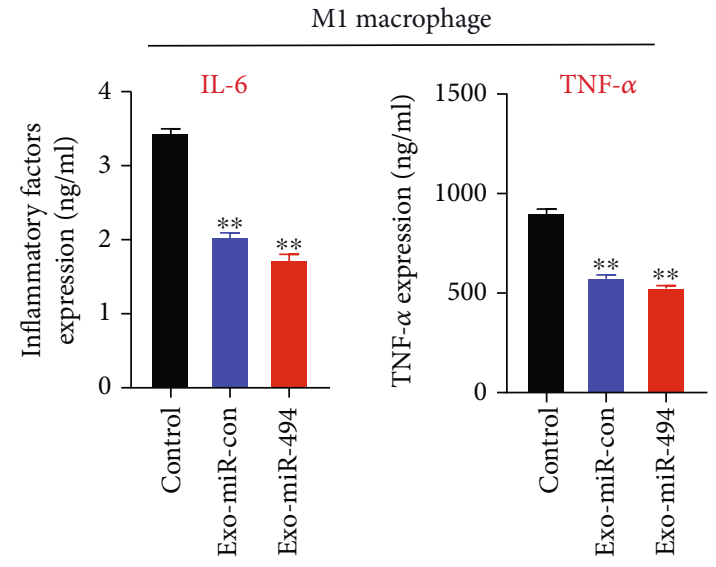

(e)

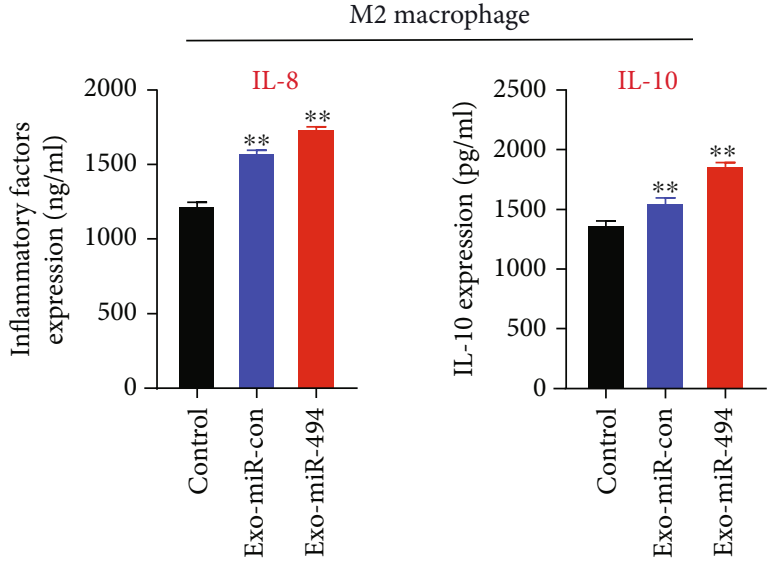

(f)

FIGURE 4: Therapeutic effect of Exo ${ }^{\text {miR-494 }}$ on DRGs and NR8383 macrophages in vitro. (a) Cell survival of DRGs was measured by AOEB staining in Control, Exo-miR-con, and Exo-miR-494 groups (the living cells are green and the dead cells are red). (b) Cell viability by cell counting in the Control, Exo-miR-con, and Exo-miR-494 groups. $n=3$, data are represented as mean \pm SD, ${ }^{*} p<0.05$ and ${ }^{* *} p<0.01$ vs. Control. (c) Western blot analysis of apoptosis-related proteins (Caspase-3, Bax, and Bcl-2) at $24 \mathrm{~h}$ in the Control group, Exo-miR-con group, and Exo-miR-494 group. (d) Relative density of Caspase-3, Bax, and Bcl-2. $n=6$, data are represented as mean $\pm \mathrm{SD}$, ${ }^{* *} p<0.01$ vs. Control. (e) ELISA analysis for secretion of M1 polarization-related proinflammatory cytokines (IL-6, TNF- $\alpha$ ) and (f) M2 polarization-related proinflammatory cytokines (IL-8, IL-10) in LPS/hypoxia-treated NR8383 macrophages at $48 \mathrm{~h}$ after ExomiR-494, ExomiR-con, and PBS administrations. $n=3$, data are represented as mean $\pm \mathrm{SD},{ }^{* *} p<0.01$ vs. Control.

consistent with existing research results (Figure 5(a)) [26], that is, exosomes injected into the body were mainly phagocytized by mononuclear in the liver. The results of $24 \mathrm{~h}$ showed that the fluorescence intensity of all Exo ${ }^{\text {miR-494 }}$ decreased significantly, demonstrating that Exo ${ }^{\mathrm{miR}-494}$ were absorbed by liver cells and nerve cells in the injured area (Figure 5(a)).

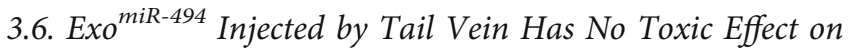
the Liver. According to the fluorescence images of animals (Figure 5(a)), we know that most of Exo ${ }^{\text {miR-494 }}$ converges to the lung and liver, so we conducted a toxicity test on healthy rats after administration of Exo ${ }^{\mathrm{miR}-494}$. The HE staining results showed that the lung and liver of the ExomiR-494 group showed a normal shape and contour, without obvious signs of injury (Figures 5(b) and 5(c)). Furthermore, the activities of enzymes closely related to liver function were detected. The results demonstrated that the activities of LDH, SOD, and ALT in rat liver after $\mathrm{Exo}^{\mathrm{miR}-494}$ treatment were similar to those in healthy rats, indicating that the exosome has no toxic side effects on rat liver, which is an ideal miRNA vector (Figure 5(d)).

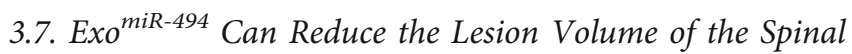
Cord. Next, we evaluated the injury volume of the transverse section of the spinal cord by HE staining. At day 28 postSCI, the lesion center of the Control group often contained typical vacuoles with irregular shape (Figure 6(a)). Compared with the Control group, the central contour of ExomiR-494 was more complete, the shape was more normal, and the damage volume was smaller (Figure 6(a)). In addition, the damage degree of the Exo-miR-con group was between that of the Control group and the Exo-miR-494 group (Figure 6(a)).
3.8. Exo ${ }^{\text {miR-494 }}$ Promotes the Regeneration of Neurofilament in Spinal Cord Injury Rats. To determine the effect of Exo $^{\text {miR-494 }}$ on neurofilament regeneration, NF-H and GFAP double-labeled immunofluorescence were employed to evaluate neurofilament regeneration and astrocyte activation 4 weeks after injury and as revealed from the results (Figure 6(b)). As a result, considerable active astrocytes were recruited and the neurofilament disintegrated in the Control group (Figure 6(b)), and the NF-positive cells in the ExomiR-494 group were significantly higher than those in the Control group and the Exo-miR-con group (Figure 6(c)); also, the GFAP-positive cells were lower than in the Control group and the Exo-miR-con group (Figure 6(d)). Thus, the Exo-miR-494 group rats showed significantly increased neurofilament and decreased astrocytes (Figure 6(d)).

At the same time, after 28 days of injury, we measured the expression of GAP-43 (Figure 6(e)). As a result, after injury, GAP-43 was upregulated in all three groups except the Sham group (Figure 6(f)). Among them, the upregulation was the highest in the Exo-miR-494 group, up to $110 \pm 4.0 \%$, and there was also a certain increase in the Exo-miR-con group (Figure 6(f)), which was derived from simple exosomes and promoted the increase of GAP-43.

\subsection{Exo ${ }^{\text {miR-494 }}$ Promotes the Recovery of Behavioral Function} in Rats with Spinal Cord Injury. Lastly, we evaluated the behavioral function of rats. To determine the effect of $\mathrm{Exo}^{\mathrm{miR}-494}$ on the recovery of the motor function of all rats, the $\mathrm{BBB}$ score was used to evaluate the locomotor capacity of all rats at each time point after injury, and as revealed from the results, the BBB scores of rats in each group increased gradually with time due to SCI rats also having a certain self-restoring capacity (Figure 7(a)). On day 28, both Exo-miR-494 group and Exo-miR-con group 


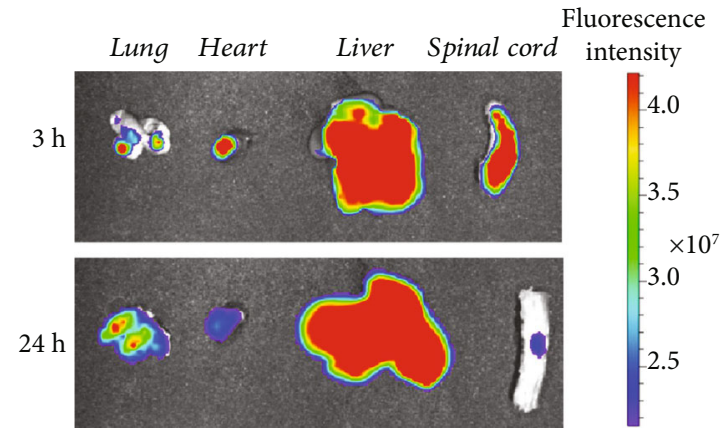

(a)
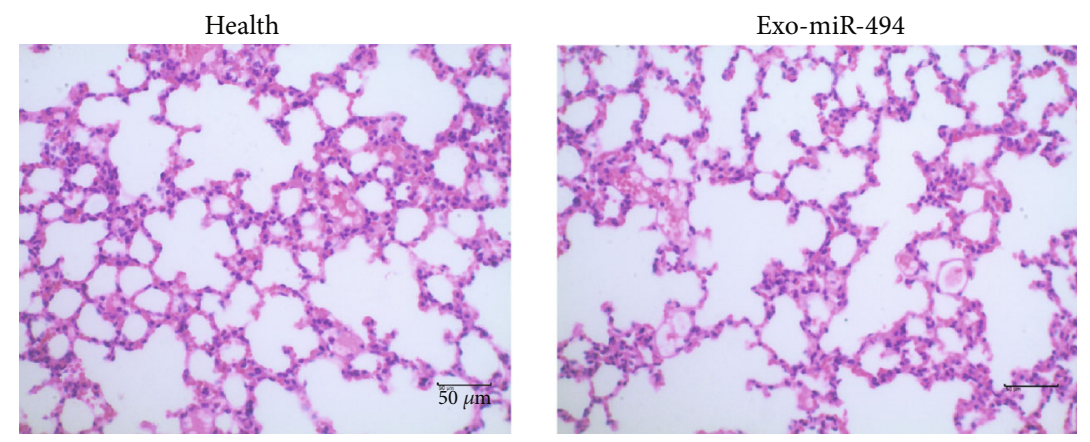

(b)
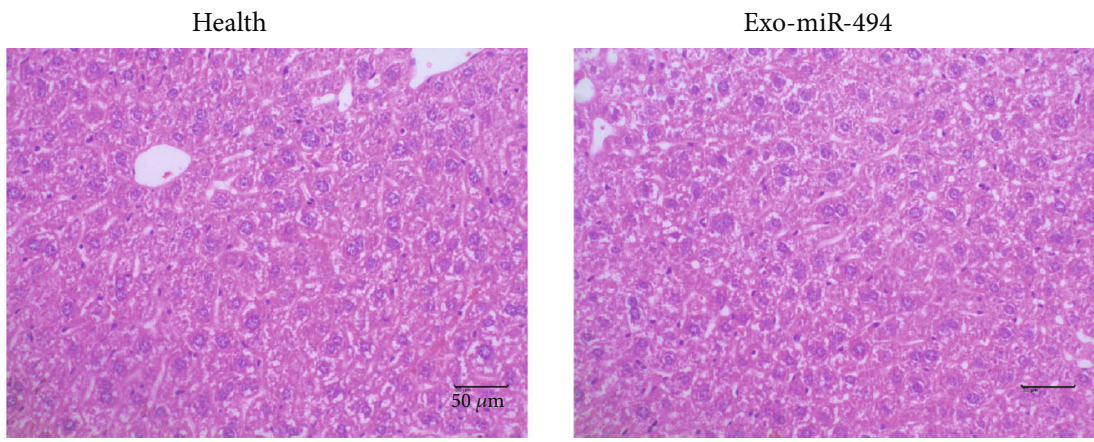

(c)

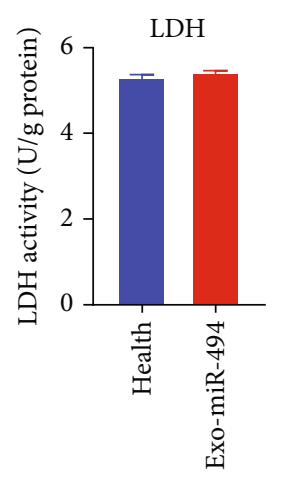

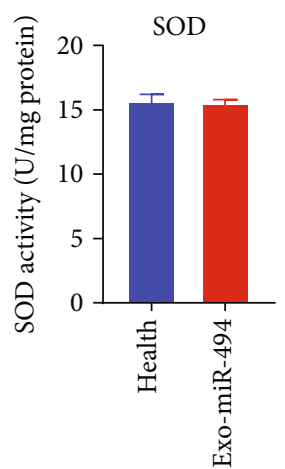

(d)
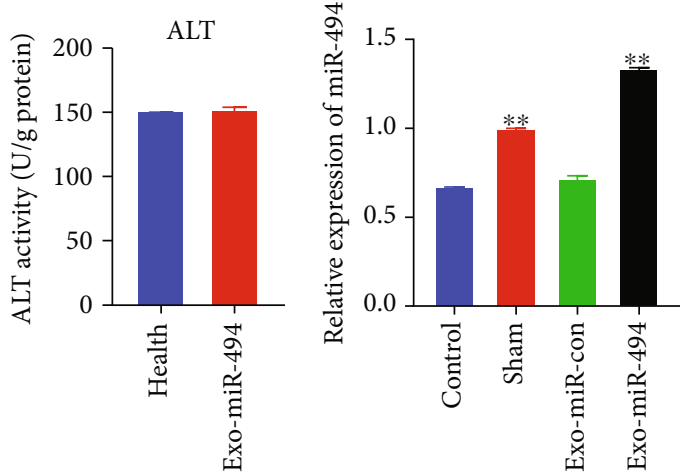

(e)

FIGURE 5: Therapeutic effect of Exo ${ }^{\mathrm{miR}-494}$ on spinal cord injury in vivo. Rats were classified into Control group, Sham group, Exo-miR-con group, and Exo-miR-494 group. (a) Fluorescence imaging of organs of Exo-miR-494 group rats at $3 \mathrm{~h}$ and $24 \mathrm{~h}$ after intravenous injection was illustrated. (b) HE staining of lung in the health group and Exo-miR-494 group. The rats in the health group were fed normally without any injury or treatment. Scale bar $=50 \mu \mathrm{m}$. (c) HE staining of liver in health group and Exo-miR-494 group. Scale bar $=50 \mu \mathrm{m}$. (d) Enzyme activities of LDH, SOD, and ALT in the liver of health group and Exo-miR-494 group rats. $n=6$. (e) The relative expressions of miR-494 in injured spinal cord at $48 \mathrm{~h}$ after Exo ${ }^{\text {miR-494-con }}, E^{2}{ }^{\text {miR-494 }}$, and PBS treatment by RT-PCR. $n=6$, data are represented as mean \pm SD, ${ }^{* *} p<0.01$ vs. Control. 

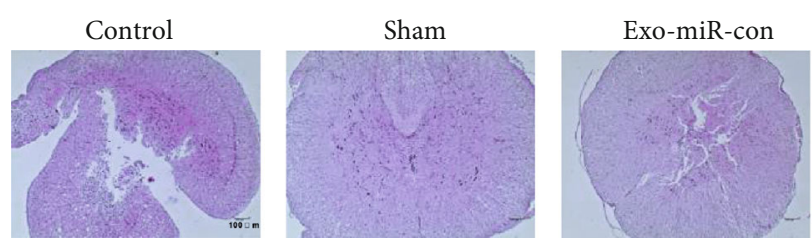

(a)
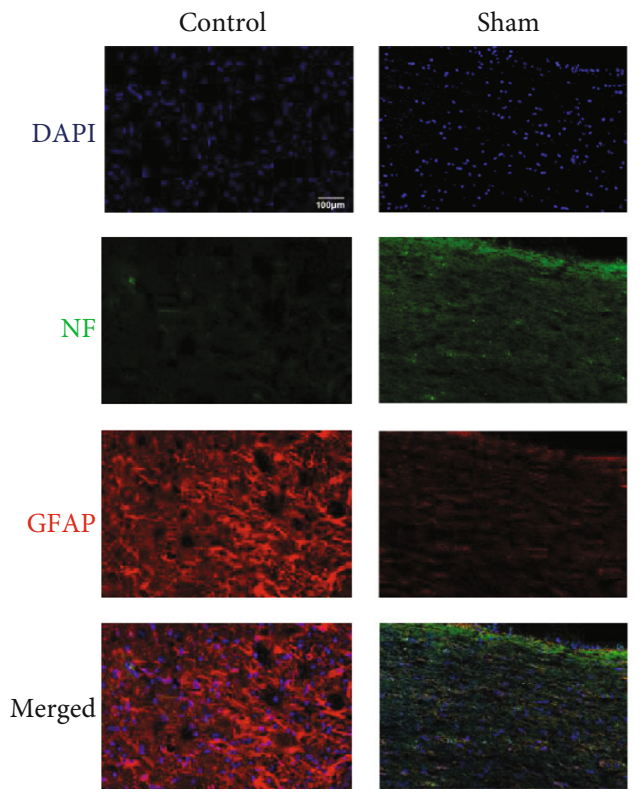

(b)
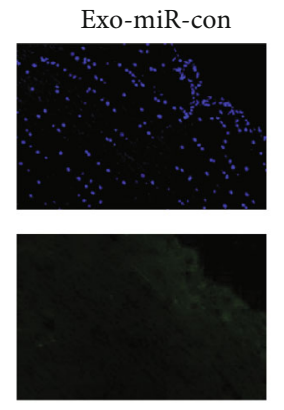

Exo-miR-494

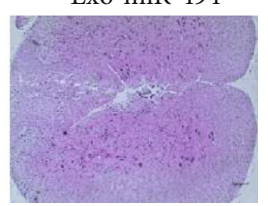

Exo-miR-494
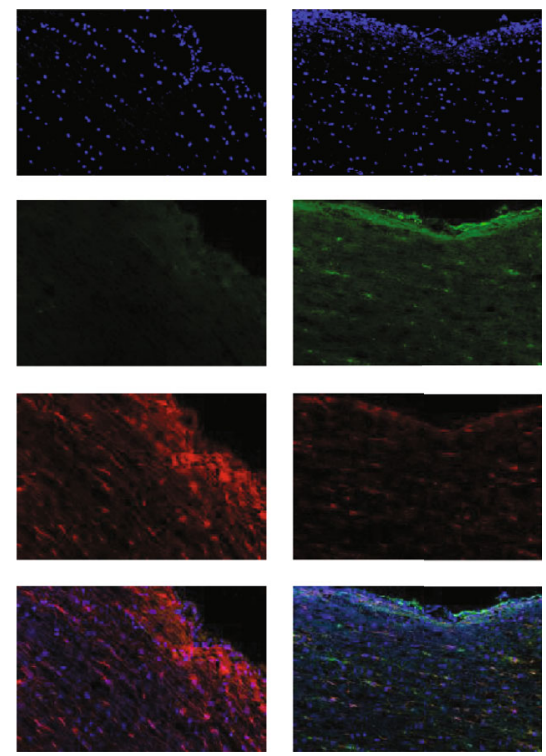

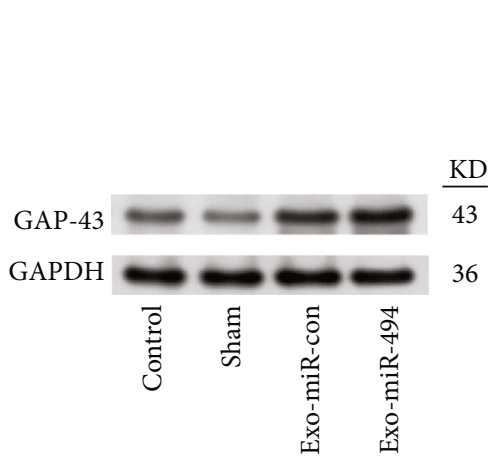

(e)

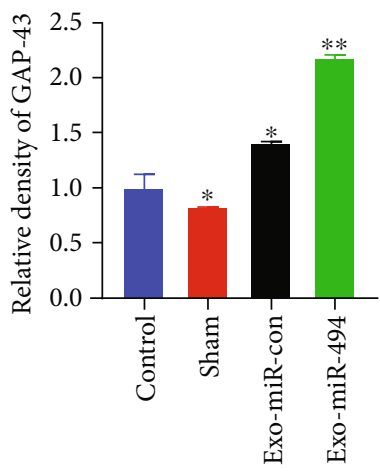

(f)

FIGURE 6: Exo ${ }^{\text {miR-494 }}$ can reduce the lesion volume of spinal cord and promote the regeneration of neurofilament. (a) On the 28th day after injury, the transverse section of spinal cord in each group was stained with HE. Scale bar $=100 \mu \mathrm{m}$. (b) Immunofluorescence images showing the expressions of neurofilament (NF, green) and astrogliosis (GFAP, red) in longitudinal section of the spinal cord lesion at 4 weeks in each group. Scale bar $=200 \mu \mathrm{m}$ or $50 \mu \mathrm{m}$. (c) Relative average fluorescence intensity of NF (\%) in each group rats by ImageJ software. The average fluorescence intensity in Sham group was set to $100 \% . n=6$, data are represented as mean $\pm \mathrm{SD},{ }^{*} p<0.05$ and ${ }^{* *} p<0.01$ vs. Control. (d) Relative average fluorescence intensity of GFAP (\%) in each group of rats. The average fluorescence intensity in Control group was set to $100 \% . n=6$, data are represented as mean $\pm \mathrm{SD},{ }^{*} p<0.05$ and ${ }^{* *} p<0.01$ vs. Control. (e) Expression of GAP-43 in rats of each group after 28 days of SCI. (f) Relative density of GAP-43 by ImageJ software. $n=6$, data are represented as mean \pm SD, ${ }^{*} p<0.05$ and ${ }^{* *} p<0.01$ vs. Control.

animals showed significant functional recovery, of which the average score of the Exo-miR-494 group was $15.8 \pm 0.7$ and that of Exo-miR-con animals was $12.7 \pm 0.5$ (Figure $7(\mathrm{a})$ ). The average score of the Control group was lower, which was only $11.2 \pm 0.65$ (Figure $7(\mathrm{a})$ ). So, the motor functional recovery of the Exo-miR-494 group was better than those of the Exo-miR-con group and Control group.
To further analyze thermal sensitivity by PWTL, on the first day before injury, all rats showed almost the same and normal PWTL, with an average of about $6.8 \pm 0.5 \mathrm{~s}$ (Figure 7(b)). One week after spinal cord injury, PWTL of rats in each group was more than $20 \mathrm{~s}$ due to severe trauma (Figure $7(\mathrm{~b})$ ). Then, the PWTL of rats in each group decreased gradually, and the most obvious decrease was in 


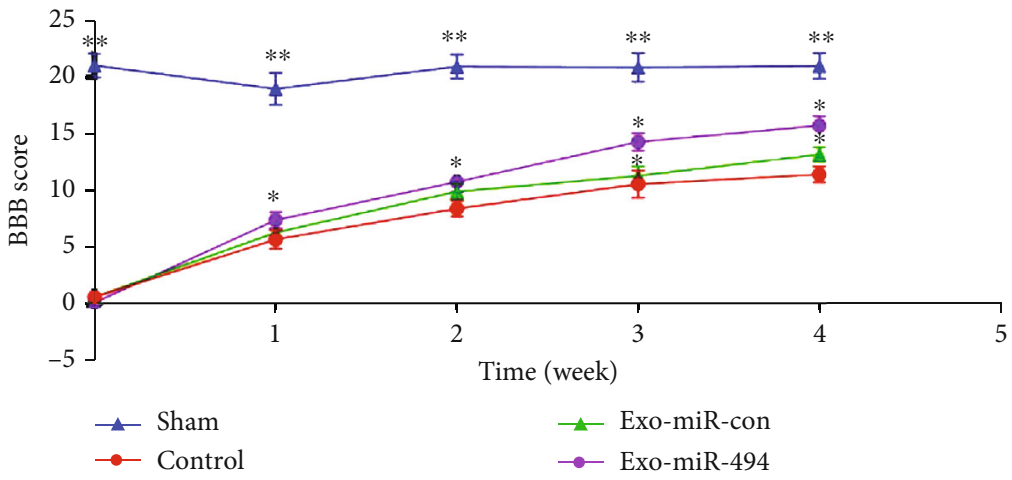

(a)

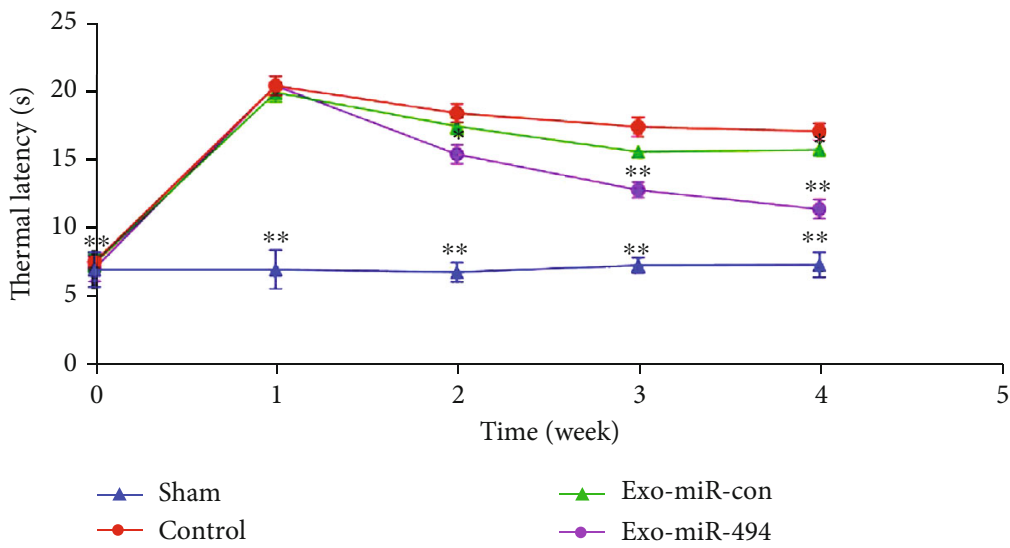

(b)

Transcranial stimulation (brain)

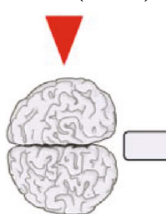

T10 spinal cord injury site

(hindlimbs)

(c)
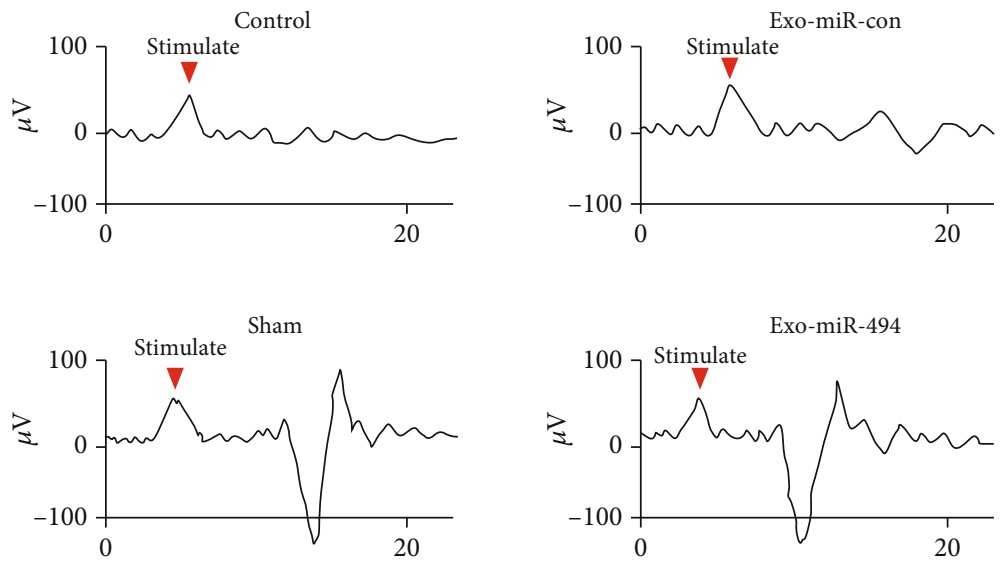

(d)

Figure 7: Continued. 


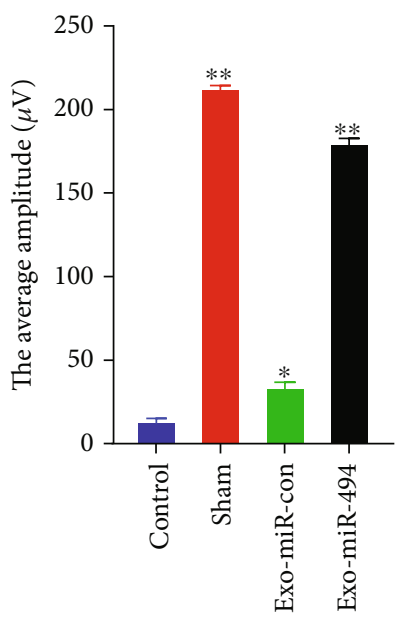

(e)

FIGURE 7: Exo ${ }^{\text {miR-494 }}$ promotes functional recovery in SCI rats. (a) BBB scores of rats in Control group, Sham group, Exo-miR-con group, and Exo-miR-494 group. $n=6$, data are represented as mean \pm SD, ${ }^{*} p<0.05$ and ${ }^{* *} p<0.01$ vs. Control. (b) The PWTL of rats in Sham group, Control group, Exo-miR-con group, and Exo-miR-494 group. $n=6$, data are represented as mean $\pm \mathrm{SD},{ }^{*} p<0.05$ and ${ }^{* *} p<0.01$ vs. Control. (c) Schematic diagram of neuroelectrophysiology. (d) MEPs of rats in Sham group, Control group, Exo-miR-con group, and Exo-miR-494 group after 4 weeks of treatment (the average of the three times was collected into a line graph, and the red arrow marked the stimulus artifact). (e) The average amplitude in Sham group, Control group, Exo-miR-con group, and Exo-miR-494 group after 4 weeks of treatment. $n=6$, data are represented as mean $\pm \mathrm{SD},{ }^{*} p<0.05$ and ${ }^{* *} p<0.01$ vs. Control.

the Exo-miR-494 group, reaching $12.7 \pm 0.4 \mathrm{~s}$ on the 28 th day, $16.5 \pm 0.8 \mathrm{~s}$ in the Exo-miR-con group, and $17.5 \pm 0.3 \mathrm{~s}$ in rats of the Control group (Figure 7(b)).

\subsection{Exo ${ }^{\text {miR-494 }}$ Promotes the Recovery of Neuroelectrophysiology} in Spinal Cord Injury Rats. 4 weeks after injury, we conducted neuroelectrophysiological experiments (Figure 7(c)). As shown in Figures 7(d) and 7(e), the MEPs in hind limbs of all rats showed that there was an obvious one-way wave in the Exo-miR-494 group, and the average amplitude $(184 \pm 4 \mu \mathrm{V})$ was significantly larger than that in the Exo-miR-con $(34 \pm 5 \mu \mathrm{V})$ or Control groups $(14 \pm 5 \mu \mathrm{V})$. Therefore, the average amplitude of MEP in the Control group was very small, which was due to the ineffective recovery of neurophysiological function.

\section{Discussion}

SCI is capable of causing severe motor and sensory disorders in patients, and no effective treatment has been applied to the clinic thus far [27]. It is increasingly evidenced that many miRNAs are involved in the pathogenesis or recovery mechanism. To be specific, miR-135a-5p can promote the recovery of spinal cord injury by mediating ROCK pathway [28], and low-level miR-130a-3p can activate the IGF-1/IGF$1 \mathrm{R}$ pathway to relieve pathological pain after spinal cord injury [29].

miR-494 refers to a tumor suppressor inhibiting the proliferation and colony formation of tumor cells [30]. miR-494 can reduce autophagy and apoptosis of PC-12 cells induced by LPS by targeting IL-13 in vitro [18]. Besides, miR-494 can also regulate the PTEN/Akt/mTOR pathway, thereby inhibiting neuronal apoptosis after SCI in rats $[22,31]$. Further- more, as reported by our previous research, SIRT1 can inhibit apoptosis after spinal cord injury via miR-494 [32].

Stem cell transplantation has been investigated in tissue regeneration for years; however, it still has some limitations $[33,34]$. Over the past few years, exosomes, a novel type of intercellular communication device, have been employed as a good biological carrier for local or systemic small RNA delivery for treating stroke or spinal cord injury and other central nervous system diseases. The mentioned exosomes play an active role in tissue regeneration, while acting as the carriers of genetic materials (e.g., mRNAs, miRNAs, and lncRNAs), which can be used as gene transfer systems for alternative cell therapy. Here, Exo ${ }^{\text {mik-494 }}$ was constructed by chemical transfection, and the therapeutic effect of ExomiR-494 on SCI was studied in depth.

First, miR-494 was loaded into the exosome effectively by chemical transfection, and it exhibited high loading efficiency, about $38 \%$ loading rate, complying with the existing results [21]. The amount of exosomes secreted in vivo is very small, so convenience was provided for the mass production of Exo ${ }^{\text {miR-494 }}$ by chemical transfection, which is considered a high loading efficiency method. Besides, exosomes exhibit excellent encapsulation and antidegradation properties, capable of effectively preventing the degradation of target miRNA in vivo environment, which provides the possibility

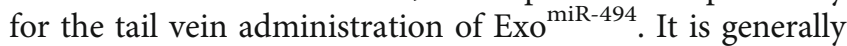
known that innate immune cells (i.e., microglia and astrocytes) and infiltrating leukocytes (i.e., macrophages and neutrophils) are activated after SCI, thereby leading to inflammatory cascade. The mentioned inflammatory cells release various neurotoxins, proinflammatory cytokines, chemokines, free radicals, excitotoxic amino acids, nitric oxide, and others, creating a very unfavorable microenvironment for the regeneration of neurons [35]. In addition, glial 
scar imposes a major obstacle on axonal regeneration. After spinal cord injury, injured fibroblasts invade the injured area and then secrete considerable extracellular matrix (e.g., type IV collagen, fibronectin, and laminin), constituting the main component of the glial scar $[35,36]$. Wang et al. reported that miR-494 can inhibit the proliferation and synaptic remodeling of spinal reactive astrocytes in SCI rats by activating the Nogo/NgR signaling pathway [19]. Reactive astrocytes are recognized as the pathological markers of central nervous system damage (e.g., Parkinson's disease, Alzheimer's disease, and spinal cord injury) [37]. Reactive astrocytes are capable of secreting considerable extracellular matrix and forming glial scar, thereby leading to limited nerve repair and axonal degeneration [38, 39]. Moreover, glial scar formation is capable of stimulating the production of GFAP, which activates the RhoA signal, causes the growth cone to collapse, and inhibits axon regeneration [40].

In this study, as revealed from NF and GFAP immunofluorescence results, Exo ${ }^{\text {miR-494 }}$ can effectively downregulate the expression of GFAP, as well as promote the increase of the neurofilament. Indeed, the mentioned positive results reveal the important role of miR-494 for treating spinal cord injury. Over time, the BBB score of Exo ${ }^{\text {miR-494 }}$ group was significantly higher than those of other groups, demonstrating that the motor function of rats had been significantly improved. In addition, to explore the effect of Exo ${ }^{\text {miR-494 }}$ on the electrophysiological function of the hind limbs of SCI rats, the electrophysiological study was conducted on the hind limbs of rats in 4 weeks. Complying with the existing results, Exo ${ }^{\text {miR-494 }}$ is capable of effectively facilitating the recovery of the neuroelectrophysiological function of SCI rats.

\section{Conclusion}

In brief, the results showed that the exosome is an excellent miRNA vector, the tail vein injection of Exo ${ }^{\text {miR-494 }}$ can upregulate the expression of miR-494, improve the local immune environment, and inhibit neuronal apoptosis and release of proinflammatory factors, thereby promoting the regeneration of the neurofilament and the recovery of behavioral function.

\section{Data Availability}

The [experimental data] data used to support the findings of this study are included within the article.

\section{Conflicts of Interest}

The authors declare that they have no conflicts of interest.

\section{Supplementary Materials}

Supplementary 1. Supplement 1: instruction manual of the GS exosome isolation reagent kit.

Supplementary 2. Supplement 2: Exo and Exo-miR-494 negative staining steps for transmission electron microscope.

Supplementary 3. Supplement 3: real-time polymerase chain reaction (RT-PCR) experimental steps.
Supplementary 4. Supplement 4: ALT test kit instructions.

Supplementary 5. Supplement 5: LDH test kit instructions.

Supplementary 6. Supplement 6: SOD test kit instructions.

\section{References}

[1] Y. Yang, Y. Fan, H. Zhang et al., "Small molecules combined with collagen hydrogel direct neurogenesis and migration of neural stem cells after spinal cord injury," Biomaterials, vol. 269, p. 120479, 2021.

[2] F. Faghihi, E. Mirzaei, J. Ai et al., "Differentiation potential of human chorion-derived mesenchymal stem cells into motor neuron-like cells in two- and three-dimensional culture systems," Molecular Neurobiology, vol. 53, no. 3, pp. 1862-1872, 2016.

[3] J. Gu, Z. Jin, C. Wang, X. F. Yan, Y. Q. Mao, and S. Chen, "Bone marrow mesenchymal stem cell-derived exosomes improves spinal cord function after injury in rats by activating Autophagy," Drug Design, Development and Therapy, vol. 14, pp. 1621-1631, 2020.

[4] G. Sun, G. Li, D. Li et al., "hucMSC derived exosomes promote functional recovery in spinal cord injury mice via attenuating inflammation," Materials Science and Engineering: C, vol. 89, pp. 194-204, 2018.

[5] P. Wu, B. Zhang, D. Ocansey, W. Xu, and H. Qian, "Extracellular vesicles: a bright star of nanomedicine," Biomaterials, vol. 269, article 120467, 2021.

[6] Y. Hu, R. Tao, L. Chen et al., "Exosomes derived from pioglitazone-pretreated MSCs accelerate diabetic wound healing through enhancing angiogenesis," Journal of Nanobiotechnology, vol. 19, no. 1, p. 150, 2021.

[7] M. Zipkin, "Big pharma buys into exosomes for drug delivery," Nature Biotechnology, vol. 38, no. 11, pp. 1226-1228, 2020.

[8] A. Alptekin, M. Khan, R. Ara et al., "Pulsed focal ultrasound as a non-invasive method to deliver exosomes in the brain/ stroke," Journal of Biomedical Nanotechnology, vol. 17, no. 6, pp. 1170-1183, 2021.

[9] Z. Huang, L. Guo, L. Huang, Y. Shi, J. Liang, and L. Zhao, "Baicalin-loaded macrophage-derived exosomes ameliorate ischemic brain injury via the antioxidative pathway," Materials Science and Engineering: C, vol. 126, article 112123, 2021.

[10] M. Beatriz, R. Vilaça, and C. Lopes, "Exosomes: innocent bystanders or critical culprits in neurodegenerative diseases," Frontiers in cell and Developmental Biology, vol. 9, article 635104, 2021.

[11] D. Dutta, N. Khan, J. Wu, and S. M. Jay, "Extracellular vesicles as an emerging frontier in spinal cord injury pathobiology and therapy," Trends in Neurosciences, vol. 44, no. 6, pp. 492-506, 2021.

[12] Y. Zhou, L. Wen, Y. Li et al., "Exosomes derived from bone marrow mesenchymal stem cells protect the injured spinal cord by inhibiting pericyte pyroptosis," Neural Regeneration Research, vol. 17, no. 1, pp. 194-202, 2022.

[13] A. Khalatbary, "Stem cell-derived exosomes as a cell free therapy against spinal cord injury," Tissue \& Cell, vol. 71, article 101559, 2021.

[14] G. Wei, G. An, Z. Shi et al., "Suppression of MicroRNA-383 enhances therapeutic potential of human bone-marrowderived mesenchymal stem cells in treating spinal cord injury via GDNF," Cellular Physiology and Biochemistry: International 
Journal of Experimental Cellular Physiology, Biochemistry, and Pharmacology, vol. 41, no. 4, pp. 1435-1444, 2017.

[15] N. Zolboot, J. X. du, F. Zampa, and G. Lippi, "MicroRNAs instruct and maintain cell type diversity in the nervous system," Frontiers in Molecular Neuroscience, vol. 14, article 646072, 2021.

[16] W. Xu, X. Wang, P. Li, K. Qin, and X. Jiang, "miR-124 regulates neural stem cells in the treatment of spinal cord injury," Neuroscience Letters, vol. 529, no. 1, pp. 12-17, 2012.

[17] Y. Yu, K. Gibbs, J. Davila et al., "MicroRNA miR-133b is essential for functional recovery after spinal cord injury in adult zebrafish," The European Journal of Neuroscience, vol. 33, no. 9, pp. 1587-1597, 2011.

[18] W. Geng and L. Liu, "miR-494 alleviates lipopolysaccharide (LPS)-induced autophagy and apoptosis in PC-12 cells bytargeting IL-13," Advances in Clinical and Experimental Medicine: official organ Wroclaw Medical University, vol. 28, no. 1, pp. 85-94, 2019.

[19] Y. Wang, J. Sun, H. Wang et al., "Effects of microRNA-494 on astrocyte proliferation and synaptic remodeling in the spinal cord of a rat model of chronic compressive spinal cord injury by regulating the Nogo/Ngr signaling pathway," Cellular Physiology and Biochemistry: International Journal of Experimental Cellular Physiology, Biochemistry, and Pharmacology, vol. 48, no. 3, pp. 919-933, 2018.

[20] D. Li, P. Zhang, X. Yao et al., "Exosomes derived from miR-133b-modified mesenchymal stem cells promote recovery after spinal cord injury," Frontiers in Neuroscience, vol. 12, p. 845, 2018.

[21] F. Aqil, R. Munagala, J. Jeyabalan et al., "Milk exosomes - natural nanoparticles for siRNA delivery," Cancer Letters, vol. 449, pp. 186-195, 2019.

[22] H. Zhu, R. Xie, X. Liu et al., "MicroRNA-494 improves functional recovery and inhibits apoptosis by modulating PTEN/AKT/mTOR pathway in rats after spinal cord injury," Biomedicine \& Pharmacotherapy, vol. 92, pp. 879887, 2017.

[23] B. Xu, Y. Zhang, X. F. du et al., "Neurons secrete miR-132 -containing exosomes to regulate brain vascular integrity," Cell Research, vol. 27, no. 7, pp. 882-897, 2017.

[24] C. Lo Sicco, D. Reverberi, C. Balbi et al., "Mesenchymal stem cell-derived extracellular vesicles as mediators of antiinflammatory effects: endorsement of macrophage polarization," Stem Cells Translational Medicine, vol. 6, no. 3, pp. 1018-1028, 2017.

[25] H. Kim, H. Kumar, M. Jo et al., "Therapeutic efficacypotentiated and diseased organ-targeting nanovesicles derived from mesenchymal stem cells for spinal cord injury treatment," Nano Letters, vol. 18, no. 8, pp. 4965-4975, 2018.

[26] B. Goldie, M. Dun, M. Lin et al., "Activity-associated miRNA are packaged in Map1b-enriched exosomes released from depolarized neurons," Nucleic Acids Research, vol. 42, no. 14, pp. 9195-9208, 2014.

[27] N. Osier, V. Motamedi, K. Edwards et al., "Exosomes in acquired neurological disorders: new insights into pathophysiology and treatment," Molecular Neurobiology, vol. 55, no. 12, pp. 9280-9293, 2018.

[28] N. Wang, Y. Yang, M. Pang et al., "MicroRNA-135a-5p promotes the functional recovery of spinal cord injury by targeting SP1 and ROCK," Molecular therapy Nucleic acids, vol. 22, pp. 1063-1077, 2020.
[29] L. Yao, Y. Guo, L. Wang et al., "Knockdown of miR-130a-3p alleviates spinal cord injury induced neuropathic pain by activating IGF-1/IGF-1R pathway," Journal of Neuroimmunology, vol. 351, p. 577458, 2021.

[30] Q. Gong, Z. Shen, Z. Sheng, S. Jiang, and S. L. Ge, "Hsa-miR494-3p attenuates gene HtrA3 transcription to increase inflammatory response in hypoxia/reoxygenation HK2 cells," Scientific Reports, vol. 11, no. 1, p. 1665, 2021.

[31] S. Gu, R. Xie, X. Liu, J. Shou, W. Gu, and X. Che, "Long coding RNA XIST contributes to neuronal apoptosis through the downregulation of AKT phosphorylation and is negatively regulated by miR-494 in rat spinal cord injury," International Journal of Molecular Sciences, vol. 18, no. 4, p. 732, 2017.

[32] X. Yu, S. Zhang, D. Zhao et al., "SIRT1 inhibits apoptosis in in vivo and in vitro models of spinal cord injury via microRNA-494," International Journal of Molecular Medicine, vol. 43, no. 4, pp. 1758-1768, 2019.

[33] W. Chen, W. Liu, Y. Bai et al., "Transplantation of mesenchymal stem cells for spinal cord injury: a systematic review and network meta-analysis," Journal of Translational Medicine, vol. 19, no. 1, p. 178, 2021.

[34] H. Salehi-pourmehr, R. Rahbarghazi, J. Mahmoudi et al., "Intra-bladder wall transplantation of bone marrow mesenchymal stem cells improved urinary bladder dysfunction following spinal cord injury," Life Sciences, vol. 221, pp. 20-28, 2019.

[35] B. Lv, X. Zhang, J. Yuan et al., "Biomaterial-supported MSC transplantation enhances cell-cell communication for spinal cord injury," Stem Cell Research \& Therapy, vol. 12, no. 1, p. 36, 2021.

[36] M. Mokalled, C. Patra, A. Dickson, T. Endo, D. Y. R. Stainier, and K. D. Poss, "Injury-induced ctgfa directs glial bridging and spinal cord regeneration in zebrafish," Science, vol. 354, no. 6312, pp. 630-634, 2016.

[37] I. Baskakov, "On the reactive states of astrocytes in prion diseases,” Prion, vol. 15, no. 1, pp. 87-93, 2021.

[38] J. Satoh, M. Irino, P. Martin, R. B. Mailman, and K. Suzuki, "Neurochemical and immunocytochemical studies of catecholamine system in the brindled mouse," Journal of Neuropathology and Experimental Neurology, vol. 50, no. 6, pp. 793-808, 1991.

[39] A. Tran, P. Warren, and J. Silver, "New insights into glial scar formation after spinal cord injury," Cell and Tissue Research, 2021.

[40] H. Tsujioka and T. Yamashita, "Neural circuit repair after central nervous system injury," International Immunology, vol. 33, no. 6, pp. 301-309, 2021. 